\title{
Camera Calibration and Error Analysis Based on MATLAB Calibration Tool
}

\author{
Yan $\mathrm{Cao}^{1, \mathrm{a}^{*}}$, Jianghao $\mathrm{Fu}^{2, \mathrm{~b}}, \mathrm{Yu} \mathrm{Bai}^{3, \mathrm{c}}$ \\ ${ }^{1}$ Department of Mechanical and Electronic Engineering, Xi'an Technological University, Xi'an, \\ China \\ ajantonyz@163,b513123695@qq.com, c8640375@qq.com
}

Keywords: Camera calibration; calibration toolbox; image coordinate

\begin{abstract}
According to the calibration principle of the MATLAB calibration toolbox, the transformation relations between four coordinate systems used in camera calibration are analyzed. On this basis, MATLAB calibration toolbox is used to simulate the corner point calibration algorithm of the checkerboard, and simulation results and error analysis are given. The validity of MATLAB calibration toolbox in camera calibration and correctness of the algorithm are obtained.
\end{abstract}

\section{Introduction}

With the continuous development of computer technology, computer-supported machine vision technology is widely used in many fields, such as reverse engineering, spatial ranging, image recognition, measurement of various parts of three-dimensional [1]. Camera calibration is an important content of computer vision research ${ }^{[2]}$, whether good or bad, calibration results have direct impact on the subsequent development of the final result. At present, the calibration method is widely used with Tsai ${ }^{[3]}$ method, the zhangzheng you calibration method ${ }^{[4]}$, and Hu Zhanyi ${ }^{[5]}$ proposed orthogonal motion method based on ${ }^{[6]}$. pole has many methods, the main research contents of the current is a specific problem, using a specific accurate, fast, simple calibration method ${ }^{[7-9] .}$ MATLAB Calibration Toolbox provides a variety of calibration routines and several classical calibration methods, in addition, provides calibration target, user interface with convenient and flexible, and the toolbox $\mathrm{C}$ source code in the computer vision library opened for the second Development provides the ideal conditions. By using the calibration tool in the MATLAB toolbox, the camera can be calibrated, and the calibration tool can be used to calibrate the camera parameters quickly and efficiently and optimize the internal parameters ${ }^{[10]}$.

\section{Calibration Principle}

The basic purpose of calibration is to realize the camera calibration from world coordinates to image pixel coordinates from world coordinates to image coordinates, as shown in Fig. 1, respectively, world coordinates, camera coordinates, image coordinates and physical coordinates.

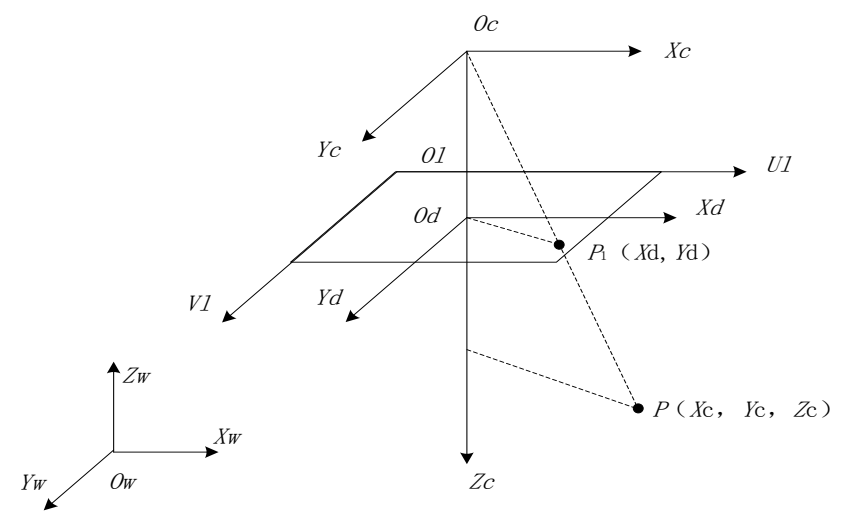

Figure 1. Camera coordinate relation 
Image coordinate system Physical coordinate: Image coordinate and physical coordinate In the same projection plane, the image coordinate is based on the upper left corner of the image as the origin, and its $(\mathrm{u}, \mathrm{v})$ respectively indicate the vertical and horizontal coordinates. The physical coordinates are based on the image center as the coordinate origin, and (x,y) represent the vertical and horizontal coordinates, respectively. Image coordinates and physical coordinates of the conversion is actually between the units of conversion. Dx and dy represent the physical dimensions of each pixel on the horizontal axis $\mathrm{x}$ and the vertical axis $\mathrm{y}$, respectively, there is a relationship between the coordinates of each pixel in the image in the $\mathrm{u}-\mathrm{v}$ coordinate system and the coordinates in the $\mathrm{x}-\mathrm{y}$ coordinate system:

$$
u=\frac{x}{d x}+u_{0}
$$

Camera coordinates: The camera coordinate system set the intersection of optical axis and mirror surface as the origin, where the $\mathrm{Xc}$ axis $\mathrm{Yc}$ axis is parallel to the $\mathrm{x}$ and $\mathrm{y}$ axes of the image coordinate system, and the Zc axis is parallel to the optical axis.

World coordinate system: The world coordinate system is introduced to describe the position of the camera. It can be used to describe the relative position and attitude of the camera. It is supposed that the homogeneous coordinates of the point $\mathrm{P}$ in the world coordinate system are (XW, YW, ZW) $(\mathrm{XC}, \mathrm{YC}, \mathrm{ZC})$ in the camera coordinates, the following relationship exists:

$$
\left[\begin{array}{c}
X_{C} \\
Y_{C} \\
Z_{C}
\end{array}\right]=\left[\begin{array}{cc}
R & T \\
0 & 1
\end{array}\right]\left[\begin{array}{c}
X_{W} \\
Y_{W} \\
Z_{W} \\
1
\end{array}\right]=M_{1}\left[\begin{array}{c}
X_{W} \\
Y_{W} \\
Z_{W} \\
1
\end{array}\right]
$$

In the above formula, $\mathrm{R}$ is an orthogonal unit matrix (also a rotation matrix), and $\mathrm{T}$ is a three-dimensional translation vector. Vector $0=(0,0,0) \mathrm{M} 1$ is the projection matrix.

Since the camera is not an ideal pinhole imaging model, there are some distortions in the manufacturing process of cameras. Three main distortion models are radial distortion, centrifugal distortion and thin prism distortion. The first type of distortion due to radial curvature only caused by radial deviation, the latter two measured both radial deviation and centrifugal deviation. The mathematical model is shown below.

$$
\left\{\begin{array}{l}
D_{x}=D_{r x}+D_{t x}+D_{p x} \\
D_{y}=D_{r y}+D_{t y}+D_{p y}
\end{array}\right.
$$

The coordinates of the coordinate system conversion relationship:

$$
\left[\begin{array}{c}
X_{C} \\
Y_{C} \\
Z_{C}
\end{array}\right]=M_{1}\left[\begin{array}{c}
X_{W} \\
Y_{W} \\
Z_{W} \\
1
\end{array}\right]+\left[\begin{array}{c}
D_{x} X \\
D_{y} Y \\
0
\end{array}\right]
$$

If it is a linear model, Dx and Dy will be 0. In the case of a nonlinear model, Dx and Dy are the sum of the distortion coefficients in the $\mathrm{X}$ direction and the $\mathrm{Y}$ direction, respectively.

\section{Camera Calibration}

The video camera used in this experiment is MV-VEM500SC, which is generally used in industry. The resolution of the output picture is $640 \times 480$. The experiment uses $7 \times 9$ template in black and white. The square of the template is $27 \mathrm{~mm} \times 27 \mathrm{~mm}$. Calibration of the internal parameters of 8 can be displayed through the Show Extrinsic camera position of the picture, and you can analyze the error by Analyze Error to determine calibration parameters are good or bad. 
(1)Run the main function calib_gui, results can be shown in Fig. 2 in a selection window.

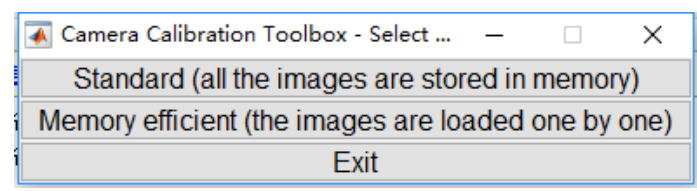

Figure 2. Select the window

Standard mode or Memory mode can be chose based on their own computer memory size, but regardless of which mode, there will appear to select the same user interface shown in Fig. 3.

\begin{tabular}{|c|c|c|c|}
\hline \multicolumn{1}{|l|}{ Camera Calibration Toolbox - Standard Version } & \multicolumn{1}{|c|}{} \\
\hline Image names & Read images & Extract grid corners & Calibration \\
\hline \hline Show Extrinsic & Reproject on images & Analyse error & Recomp. corners \\
\hline Add/Suppress images & Save & Load & Exit \\
\hline Comp. Extrinsic & Undistort image & Export calib data & Show calib results \\
\hline
\end{tabular}

Figure 3. Calibrate the main window

(2)In the main window, choose to read the picture name, and then get the required calibration of the picture.

(3)Get corners. Program running interface is shown in Fig. 4.

(4)In order to extract each corner of the map, the search window is set slightly larger, but not beyond the size of a box. Those settings are able to accurately capture the deviation from the corner, improve the accuracy of the data. After corner extraction, Calibration to calibrate the camera can be selected. When several iterations are finished, you can get the internal parameters of the camera's internal and external parameters in table 1. After obtaining the internal parameters, you can see through the Show Extrinsic calibration target relative to the camera position, as shown in Fig 5. Through the analysis of the internal parameters in Table 1, it shows that the use of calibration toolbox results is satisfactory, and the basic data to meet the experimental requirements.

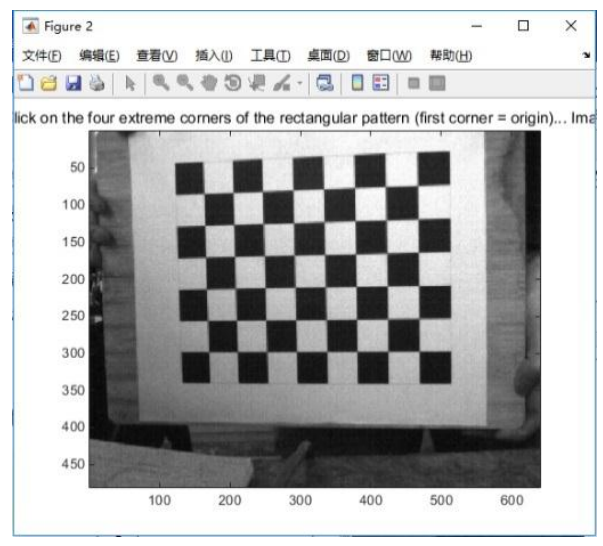

Figure 4. Program running interface

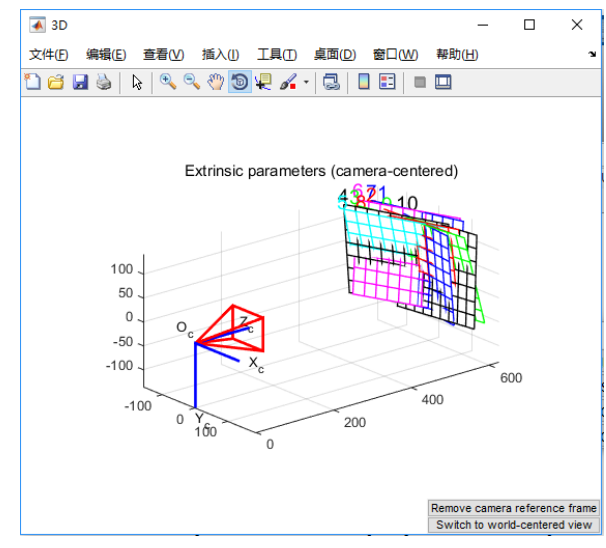

Figure 5. Calibrates the external orientation of the image

\section{Conclusion}

According to the experimental results, this method, which is a very convenient method, is not only simple, easy to implement and user-friendly, but also easy interaction, flexible setting and simple operation. Nevertheless, the method has more improved space with higher accuracy and robustness. The method is between the traditional calibration and the self-calibration, and it avoids the shortcomings of the traditional method and the high precision of the self-calibration operation. 
Table 1 Internal parameters(Unit: Pixel)

\begin{tabular}{|c|c|c|c|c|}
\hline $\begin{array}{l}\text { Parameter } \\
\text { name }\end{array}$ & Interpretation & $\begin{array}{l}\text { Parameter } \\
\text { symbol }\end{array}$ & $\begin{array}{l}\text { Calibration } \\
\text { value }\end{array}$ & Deviation \\
\hline Focal & focal length & fc & [931.73377 & {$[8.80021$} \\
\hline length & & & 933.09849] & $8.82507]$ \\
\hline Principal & the main point & $\mathrm{cc}$ & [321.94125 & {$[5.07191$} \\
\hline point & & & 237.17153] & 4.09843] \\
\hline Skew & tilt factor & alpaca & {$[0.0000$} & {$[0.0000$} \\
\hline coefficient & & & $0.00000]$ & $0.00000]$ \\
\hline \multirow{5}{*}{$\begin{array}{l}\text { Distortion } \\
\text { coefficients }\end{array}$} & \multirow{5}{*}{ distortion } & \multirow{5}{*}{$\mathrm{kc}$} & {$[-0.13814 ;$} & {$[0.01729$; } \\
\hline & & & 0.22752 & 0.11662 \\
\hline & & & $-0.00331 ;$ & 0.00108 \\
\hline & & & 0.00371 & 0.00120 \\
\hline & & & $0.00000]$ & $0.00000]$ \\
\hline
\end{tabular}

\section{Acknowledgments}

This paper is supported by both Shaanxi Provincial Department of Education special research projects under Granted NO.16JK1388 and the Key Laboratory of Special Processing NO.15JS041. The authors hereby thank both Education Department of Shaanxi Province and the Key Laboratory of Special Processing for the financial aids.

\section{References}

[1] L. Chen. Research on camera calibration based on MATLAB [J]. Science and Technology Innovation and Application, 2016, (3): 44.

[2] Y.Y. Xu, Min Zou, Xinjiang He. Research and Implementation of Camera Calibration [J]. Industrial Control Computer, 2015, (12): 23-24.

[3] Tsai R Y. A Versatile Camera Calibration Technique for High-Accuracy 3D Machine vision Metrology Using Off -the-Shelf TV Cameras and Lenses[J].IEEE Journal of Robotics Automation, 1987,3(4):323-344.

[4] Z.Y Hu, F.C Wu. A Review on Some Active Vision Based Camera Calibration Technique [J]. Chinese Journal of Computers, 2002, 25 (11):1149-1156.

[5] Schreyer H W, Garcia D, Sutton M A. Advances in Light Microscope Stereo Vision [J]. Experimental Mechanics, 2004, 44(3):278-288.

[6] Z.Y. Xu, C.L. Li, Q.C. Su. C. Ning, Camera Internal Parameter Calibration and Optimization Method Based on Genetic Algorithm [J]. Journal of JiLin University, 2014, 52(6):1267-1270.

[7] R. Y. Tsai, An efficient and accurate camera calibration technique for 3D machine vision [A].Proceedings of IEEE Conference on Computer Vision and Pattern Recognition[C]. Miami Beach, FL, 1986, 364-374.

[8] R.Y. Tsai. A versatile camera calibration technique for high-accuracy 3D machine vision metrology using off-shelf TV cameras and lenses [J]. IEEE Journal of Robotics and Automation, 1987, RA-3(4): 323-340.

[9] Z. G, Jin., J.D. Wei, G.Y. Zang and so on. A New Camera Calibration Method Based on LCD [J]. Remote Sensing Applications, 2008, (1): 87-90.

[10] Y.Q. Sun. X.P. Ji. Application of Matlab Calibration Toolbox in Camera Calibration [J]. Journal of Taiyuan Science and Technology, 2010, (03):99-100. 